\title{
Transient Ischemic Dilation
}

National Cancer Institute

\section{Source}

National Cancer Institute. Transient Ischemic Dilation. NCI Thesaurus. Code C94996.

The ratio of the left ventricular cavity volume during stress to the left ventricular cavity volume at rest. 